\title{
Braquiterapia guiada por imagen
}

\section{Image-guided brachytherapy}

\author{
E. Villafranca, P. Romero, A. Sola, G. Asín, M. Rico, M.T. Vila
}

\section{RESUMEN}

La braquiterapia consiste en la administración de radiación en contacto íntimo con el tumor, con una baja exposición de los tejidos sanos circundantes. Empezó a utilizarse a comienzos del siglo XX y desde entonces ha ido desarrollándose: diferentes radioisótopos, sistemas de tratamiento a distancia, programas informáticos que permiten un cálculo individualizado de la dosis.

Los cambios en los últimos años dentro de la braquiterapia han afectado a dos aspectos. En primer lugar, la incorporación de las técnicas de imagen como la ecografía, la tomografía computarizada (TC) y la resonancia magnética (RM), imprescindibles para el diagnóstico y la estadificación tumoral. Su utilización mientras se realiza el implante ayuda a guiarlo y realizarlo con mayor precisión. En segundo lugar, la utilización de TC, RM y ecografía permiten mejorar la cobertura del tumor o reducir la dosis a los órganos sanos. Se utilizan dentro de sistemas de planificación inversa, que realizan el cálculo de dosis a partir de las recomendaciones de las dosis a administrar al tumor y a los órganos sanos. En estos programas de planificación es posible hacer los cálculos con mucha rapidez, teniendo en cuenta la colocación en cada momento de la fuente. Esta técnica, llamada planificación en tiempo real, empieza a mostrar ventajas en el tratamiento de los cánceres de próstata.

La incorporación de las técnicas de imagen y las mejoras en los sistemas de cálculo han hecho que en la actualidad la braquiterapia juegue un papel importante en el tratamiento del cáncer de próstata, cérvix, mama, tumores de cabeza y cuello, bronquio o esófago.

Palabras clave. Braquiterapia guiada por imagen. Planificación en tiempo real. Cáncer de próstata. Cáncer de mama. Cáncer de cérvix.

\begin{abstract}
Brachytherapy consists in the administration of radiation in intimate contact with the tumour, with a low exposure of neighbouring healthy tissues. Its use began in the early XX century and it has developed since then: different radioisotopes, systems of remote treatment, computer programs making individual dose calculation possible.

In recent years there have been changes affecting two aspects of brachytherapy. In the first place, the incorporation of imaging techniques such as echography, computerised tomography (CT) and magnetic resonance (MR), indispensable for diagnosis and tumoural staging. Their use when the implant is being done helps in guiding and carrying out the operation with greater precision. In the second place, the use of CT, MR and echography makes better coverage of the tumour possible, or reduces the dose to healthy organs. They are used in inverse planning systems, which carry out dose calculation on the basis of the doses to be administered to the tumour and healthy organs. In these planning programs it is possible to make calculations more rapidly, taking account of the placement of the source at each moment in time. This technique, called real-time planning, is starting to show advantages in the treatment of prostate cancer.

Incorporation of imaging techniques and improvements in calculation systems mean that brachytherapy is currently playing an important role in treating cancer of the prostate, cervix, breast, head and neck tumours, bronchial tubes or oesophagus.
\end{abstract}

Key words. Image guided brachytherapy. Real-time planning. Prostate cancer. Breast cancer. Cervical cancer.

An. Sist. Sanit. Navar. 2009; 32 (Supl. 2): 51-59

Servicio de Oncología Radioterápica. Hospital de Navarra. Pamplona.

\author{
Correspondencia \\ Elena Villafranca Iturre \\ Servicio de Oncología Radioterápica \\ Hospital de Navarra. \\ Irunlarrea, 3 \\ 31008 Pamplona. Navarra \\ Tfno. 848428474 \\ E-mail: evillafi@cfnavarra.es
}




\section{INTRODUCCIÓN}

La braquiterapia es el tratamiento del cáncer más antiguo. En 1913 se realizó el primer tratamiento de un cáncer de cérvix con radium ${ }^{1}$. Esta técnica consiste en la administración de radiación en contacto íntimo con el tumor, con una baja exposición de los tejidos sanos circundantes. Por tanto conlleva evidentes ventajas terapéuticas, por la posibilidad de un tratamiento preciso e intenso con menores daños a los tejidos circundantes.

Inicialmente este tratamiento se aplicaba de forma manual, con la colocación de la fuente utilizando las manos y con la guía del tacto y los ojos. Esto se asociaba a localizaciones tumorales muy limitadas por su acceso y a una exposición a radiaciones ionizantes por parte del personal implicado en los tratamientos. Un paso importante fue la aparición en los años 50 de los equipos de carga diferida remota, que permiten la colocación del material radiactivo de una manera automática y controlada por ordenadores a distancia, evitando la exposición radiológica del personal médico ${ }^{2}$.

A lo largo del siglo XX también fueron variando los radioisótopos. Inicialmente se utilizó el radium, hoy prácticamente retirado del uso clínico por sus riesgos radiobiológicos, y se utiliza predominantemente ${ }^{137} \mathrm{Cs},{ }^{192} \mathrm{Ir},{ }^{125} \mathrm{I},{ }^{103} \mathrm{Pd}$ y otros en menor medida con ${ }^{241} \mathrm{Am},{ }^{60} \mathrm{Co},{ }^{75} \mathrm{Se} . .$.

Estos radioisótopos presentan diferentes tasas de dosis, o sea de cantidad de radiación que emiten por unidad de tiempo. Así se distinguen fundamentalmente: baja tasa de dosis (BTD) con una exposición de 0,4-2 Gy/hora y alta tasa de dosis (ATD) en los que es mayor de 12 Gy/hora ${ }^{3}$. Inicialmente todos los equipos de braquiterapia eran de BTD; hoy se mantienen en algunos centros, con equipos de ${ }^{137} \mathrm{Cs}$ e ${ }^{192} \mathrm{Ir}$ principalmente. En este caso, los tratamientos se administran de manera continuada por periodos que varían de horas a días, según la dosis necesaria. Ello obliga al aislamiento radiológico de los pacientes en habitaciones blindadas. También pertenece al grupo de BTD los tratamientos permanentes con semillas de ${ }^{125} \mathrm{I} o{ }^{103} \mathrm{Pd}$, utilizados principal- mente en el cáncer de próstata; en este caso la bajísima tasa de dosis de $8 \mathrm{cGy} / \mathrm{h}$ y de energía de $27 \mathrm{kev}$ permite el tratamiento de los pacientes sin aislamiento radiológico.

Los equipos de ATD, principalmente de ${ }^{192}$ Ir, han modificado la administración de los tratamientos, convirtiéndolos en tratamientos fraccionados de manera similar a la radioterapia externa (RTE). Requieren aislamiento radiológico de los pacientes tan sólo los breves minutos que dura el tratamiento, y en algunos casos permite su administración de manera ambulatoria. Además, esta fuente tiene un tamaño menor que las previas de ${ }^{137} \mathrm{Cs}$, con un diámetro alrededor de 1 milímetro, lo que facilita el acceso de la fuente a través de catéteres muy finos en cavidades pequeñas, como es el tratamiento de las lesiones endobronquiales, esofágicas o de vía biliar.

En las últimas dos décadas, la braquiterapia se ha sumado progresivamente al desarrollo tecnológico experimentado por la RTE. Son dos las áreas de mayor avance: la incorporación de técnicas de imagen, el desarrollo de los sistemas de planificación de dosis con planificación inversa y el cálculo de dosis en tiempo real ${ }^{4,5}$.

\section{SITUACIÓN ACTUAL DE LA BRAQUITERAPIA}

Los avances de la braquiterapia han afectado a la incorporación de las técnicas de imagen como la ecografía, la tomografía computarizada (TC) y la resonancia magnética (RM). Es evidente que hoy estas técnicas resultan imprescindibles para el diagnóstico y estadificación tumoral. Pero su utilidad viene de poder utilizarlos mientras se realiza el implante para poder guiarlo y realizarlo con mayor precisión. Estas técnicas permiten un mejor conocimiento de la relación entre el aplicador y el tumor, pero también con los órganos sanos.

Además, los sistemas de planificación actuales de braquiterapia han incorporado estas técnicas de imagen. Hasta hace unos pocos años, la planificación y dosimetría únicamente se basaba en la colocación geométrica de la fuentes y en reglas fijas, derivadas de la reglas del "Sistema de Man- 


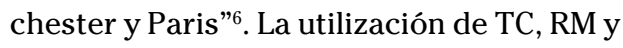
ecografía permiten realizar optimizaciones al cálculo clásico para mejorar la cobertura del tumor o reducir la dosis a los órganos sanos, así como disminuir las inhomogeneidades de este tratamiento que se asocian con mayor toxicidad. Para ello, se han incluido, al igual que en RTE, sistemas de planificación inversa, que realizan el cálculo de dosis a partir de las recomendaciones indicadas por el médico de las dosis a administrar al tumor y a los órganos sanos. Otro paso más de estos sistemas de planificación es la posibilidad de hacer los cálculos con mucha rapidez, teniendo en cuenta la colocación en cada momento de la fuente. Esta técnica, llamada, planificación en tiempo real, empieza a mostrar ventajas en el tratamiento de algunos tumores.

La incorporación de las técnicas de imagen en braquiterapia ha hecho mejorar los resultados de los tratamientos en diversas áreas: cáncer de próstata, ginecológicos, mama, cabeza y cuello, endobronquiales...

Se presentarán algunos datos de esta revisión así como las guías que los principales grupos europeo (GEC-ESTRO) y ame- ricano (ABS) han redactado para incluirlas en las diversas localizaciones tumorales.

\section{CÁNCER DE PRÓSTATA}

Los implantes permanentes de semillas radiactivas se empezaron a utilizar en el tratamiento del cáncer de próstata en los años 60. Entonces se realizaban a través de un acceso quirúrgico suprapúbico. Los resultados eran malos con control tumoral ${ }^{7}$ del $44 \%$ a 10 años y fue reemplazado por la prostatectomía en muchos centros.

Sin embargo, desde los años 80 se incorporó la utilización de ecografía endorrectal para guiar la colocación del implante vía transperineal. La ecografía permite ver la colocación de las semillas dentro de la próstata. La utilización de planificadores en tiempo real guían la colocación de las semillas con visión directa de su posición dentro de la próstata y su relación con órganos sanos como la uretra o el recto, donde la dosis debe ser inferior. A través de estos sistemas se obtiene información instantánea de la distribución de dosis en cada momento y es posible hacer las modificaciones necesarias para su optimización (Fig. 1).

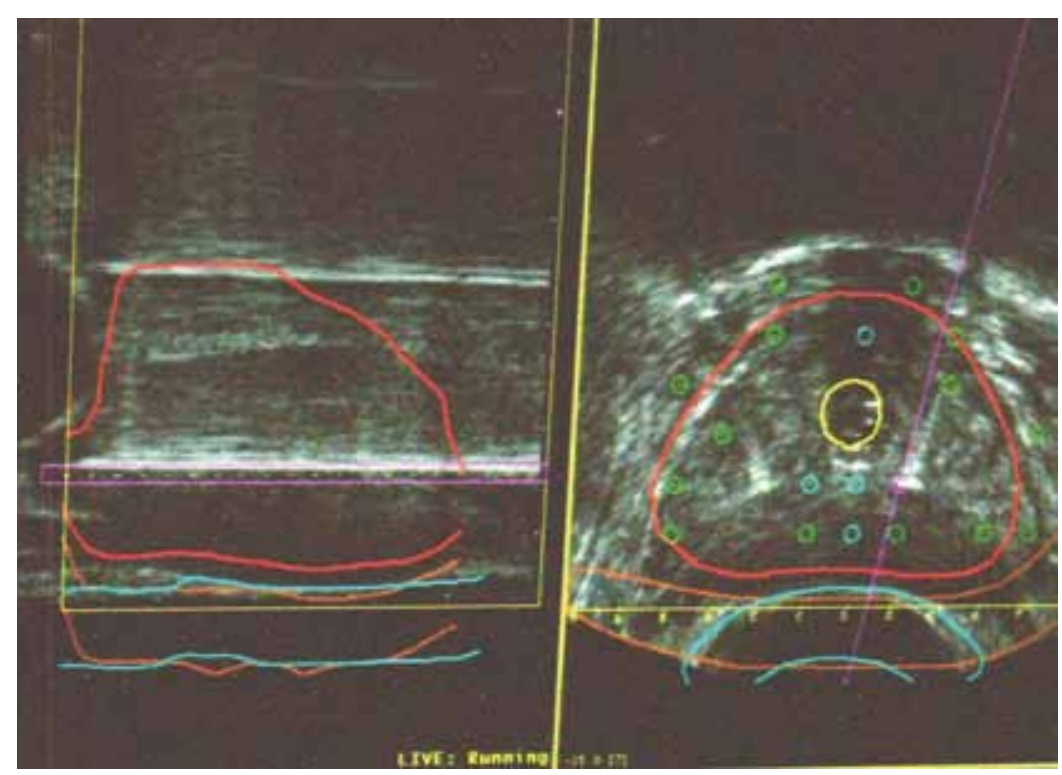

Figura 1. Implante de semillas de ${ }^{125} \mathrm{I}$ en cáncer de próstata. Planificación intraoperatoria en tiempo real con imagen guiada de ecografía. 
$\mathrm{La} \mathrm{ABS}^{8}$ introdujo en sus guías la sugerencia de utilizar estos sistemas de planificación en tiempo real respecto a los sistemas de planificación previa al implante y posteriormente ha sido suscrito por la GEC-ESTRO $^{9-10}$. Es estas últimas guías se dan además algunas recomendaciones basadas en imagen tridimensional sobre las dosis a administrar al tumor y a los órganos sanos, especialmente a la uretra y el recto, ya que está en fase de estudio las dosis relacionadas con la toxicidad en otras localizaciones como el cuello vesical, los pedículos vasculonerviosos o el bulbo peneano.

Esto ha modificado los resultados en los últimos años. Una reciente revisión multicéntrica americana mostró un control local tumoral del 87-90 \% en pacientes con cáncer de próstata de bajo riesgo ${ }^{11}$, siendo hoy un tratamiento estándar en estos pacientes $^{12}$. En pacientes con cáncer de próstata de riesgo intermedio y alto su utilización se asocia a la administración combinada con RTE, porque en estos estadios existe riesgo de extensión tumoral extraprostática ${ }^{13}$. Se han realizado análisis de calidad de vida entre pacientes tratados con braquiterapia y RTE o cirugía, con ventajas de la primera en menores cifras de incontinencia urinaria, impotencia y toxicidad rectal, aunque si mayores molestias uretrales ${ }^{14}$.

Se empieza a disponer de algunos datos clínicos de que la planificación intraoperatoria mejora los resultados, especialmente disminuyendo la toxicidad urinaria y rectal aguda y tardia ${ }^{15}$.

En los últimos 15-20 años se ha comenzado a utilizar la braquiterapia prostática a través de tratamientos con ATD. Se realiza habitualmente de manera combinada con RTE y a través de 1 a 4 fracciones. A estos tratamientos, además de la utilización de la ecografía endorrectal, se une la RM y sus técnicas de espectroscopia o difusión. Permiten una mejor definición del tumor en la próstata y está en estudio el impacto clínico de la utilización de dosis muy altas en estas regiones dentro de la misma próstata. Hay datos ya de la utilización de ATD en combinación con RTE en cáncer de riesgo intermedio y alto, con control local del $80 \%$ a 5 años, con baja toxicidad rectal y ure$\operatorname{tral}^{16,17}$.

\section{CÁNCER GINECOLÓGICO}

El tratamiento de tumores ginecológicos con braquiterapia se centra principalmente en los carcinomas de cérvix y endometrio.

En el caso del carcinoma de cérvix, la mayoría de los tratamientos de braquiterapia se realizan directamente sobre el tumor ya que éste es inoperable y se asocia a tratamientos de RTE y quimioterapia. La aplicación es endocavitaria, con la colocación de una sonda dentro del útero y de un aplicador vaginal, ya sean colpostatos o anillo, alojado en la vagina. En algunos casos de tumores muy avanzados en los que esto no puede realizarse, el implante es intersticial, con la colocación de agujas que atraviesan el cérvix y los tejidos próximos como el endometrio y los parametrios.

Estos tratamientos han sido la piedra angular de la braquiterapia, permitiendo la curación de tumores muy avanzados, algunos en estadios IIIB con extensión hasta pared pélvica, con control tumoral del 50$60 \%{ }^{18}$.

En los últimos años, se incorporó inicialmente la ecografía, que permitía una medición del tamaño tumoral, además del que se realiza con la exploración manual. Pero sobre todo ha resultado de importancia la incorporación de la RM en la última década. Ésta permite una mejor definición del tumor y también de la localización de los órganos sanos como la vejiga, el recto y el sigma ${ }^{19}$.

En el año 2005 y posteriormente en 2007, la GEC-ESTRO aconsejó la implementación progresiva de la utilización de la RM en cáncer de cérvix, y aportó recomendaciones en las dosis al tumor y órganos sanos en relación a volúmenes de estos, de nuevo basado en dosimetría tridimensional guiada por imagen ${ }^{20,21}$. Estas recomendaciones han sido también recogidas por la $\mathrm{ABS}^{22}$ (Fig. 2). 


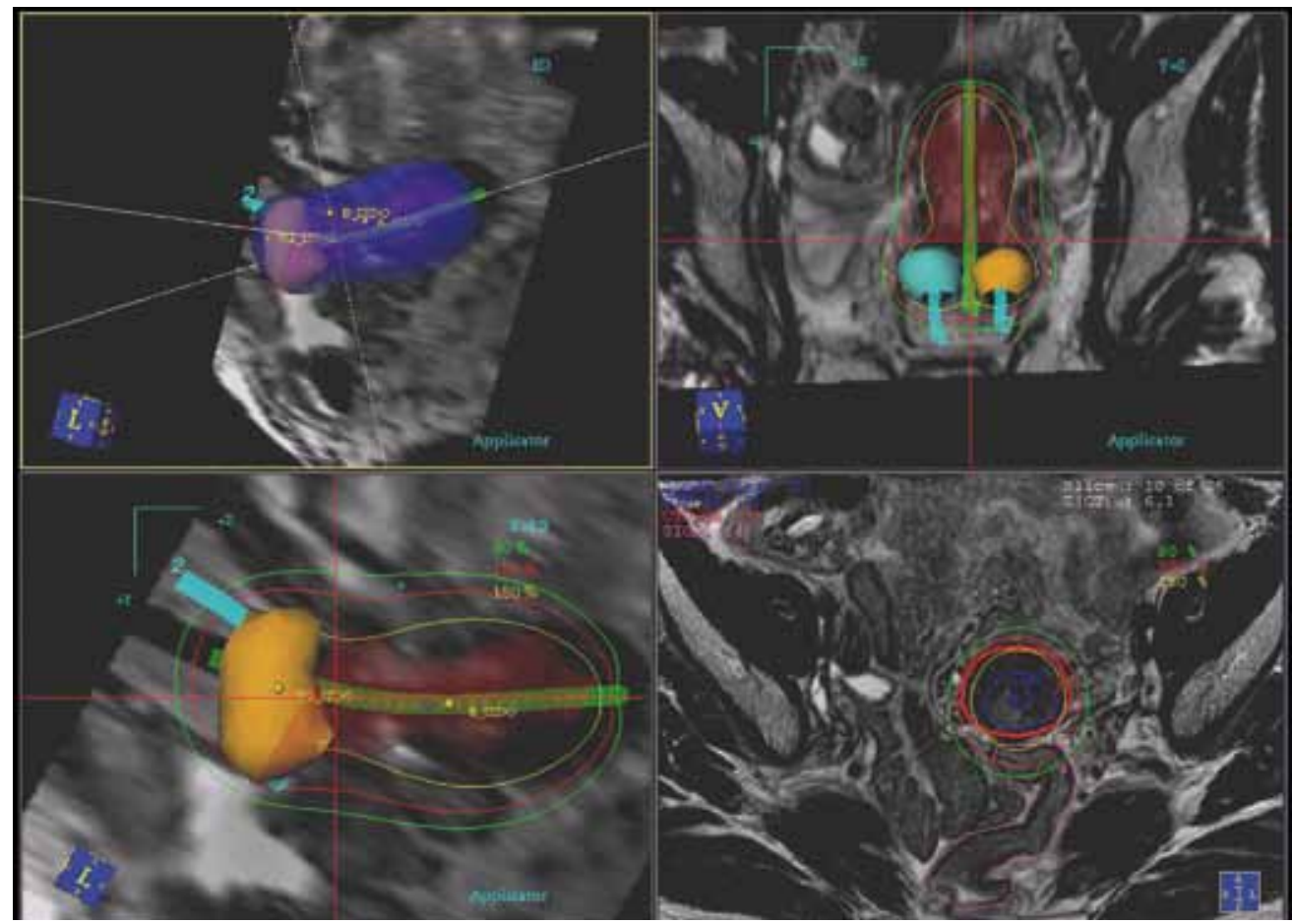

Figura 2. Implante endocavitario en cáncer de cérvix. Planificación basada en RM realizada tras la colocación de aplicador Fletcher Suit.

Los primeros datos clínicos utilizando estas guias han sido comunicados por el Hospital General de Viena y el Instituto Gustav Roussy de París. La dosis se adapta al volumen tumoral, permitiendo una escalada de dosis desde 80 a 87 GY dentro del tumor, manteniendo sin cambios las dosis en los órganos sanos. Las cifras de control tumoral son del $88 \%$ a tres años, aumentando de manera significativa en los tumores mayores de $5 \mathrm{~cm}$. La toxicidad gastrointestinal y genitourinaria severa se redujo del 10 al $3 \%$ con la incorporación de $\mathrm{RM}^{23}$. Estos datos deben reproducirse en otros centros y por ello se puso en marcha el estudio EMBRANCE, con los objetivos de apoyar la utilización de RM en braquiterapia ginecológica y obtener resultados contrastados.

\section{CÁNCER DE MAMA}

La braquiterapia ha estado incluida clásicamente como parte del tratamiento postope- ratorio del cáncer de mama. Ésta se realizaba como sobreimpresión del lecho tumoral después de la irradiación completa de la mama. Su utilidad en reducir el fallo local quedó demostrada con el ensayo de la EORTC ${ }^{24}$.

Sin embargo, en los últimos años se abre otro camino. En pacientes con tumores menores de $3 \mathrm{~cm}$, con histología de bajo grado, sin componente intraductal extenso, con baja afectación ganglionar en la linfadenectomía axilar, las tasas de fallo local son muy bajas. Existen datos de estudios fase II en los que se han analizado los resultados con irradiación parcial de la mama (IPM), realizándose ésta en la mayoría de ellos con braquiterapia. En estos estudios se analizan las recaídas después de irradiación del lecho tumoral en lugar de toda la mama. Los datos de fallo local oscilan entre $0,3-0,8 \%$ y son similares al tratamiento convencional ${ }^{25-27}$.

Por ello, se han puesto en marcha estudios fase III, de los que destacan los que 
realizan los grupos de la EORTC y RTOG, con el objetivo de confirmar estos resulta$\operatorname{dos}^{28,29}$. Incluyen pacientes con carcinoma invasivo o intraductal, con un tamaño menor o igual a $3 \mathrm{~cm}$, los márgenes quirúrgicos deben ser negativos y tener una afectación en los ganglios axilares menor de tres. El tratamiento de IPM se realiza habitualmente en una semana a diferencia de seis semanas del tratamiento convencional, lo que resulta beneficioso especialmente para pacientes con dificultades en el acceso a hospitales por un periodo largo.

En ellos, se han incluido pacientes tratadas con dos técnicas de braquite- rapia: intersticial multicanal y unicanal (MammoSite) $^{30}$. La primera consiste en cubrir el lecho tumoral con un implante de catéteres en dos o tres planos. Progresivamente se van utilizando en mayor medida los catéteres plásticos, con mejor tolerancia para las pacientes. Para un diseño adecuado del implante, éste se realiza guiado por imagen ecográfica del lecho y se utiliza la TC para la planificación y dosimetría (Fig. 3). El otro aplicador unicanal o MammoSite consiste en un catéter único flexible asociado a un balón hinchable, que se coloca de manera intra o postoperatoria.

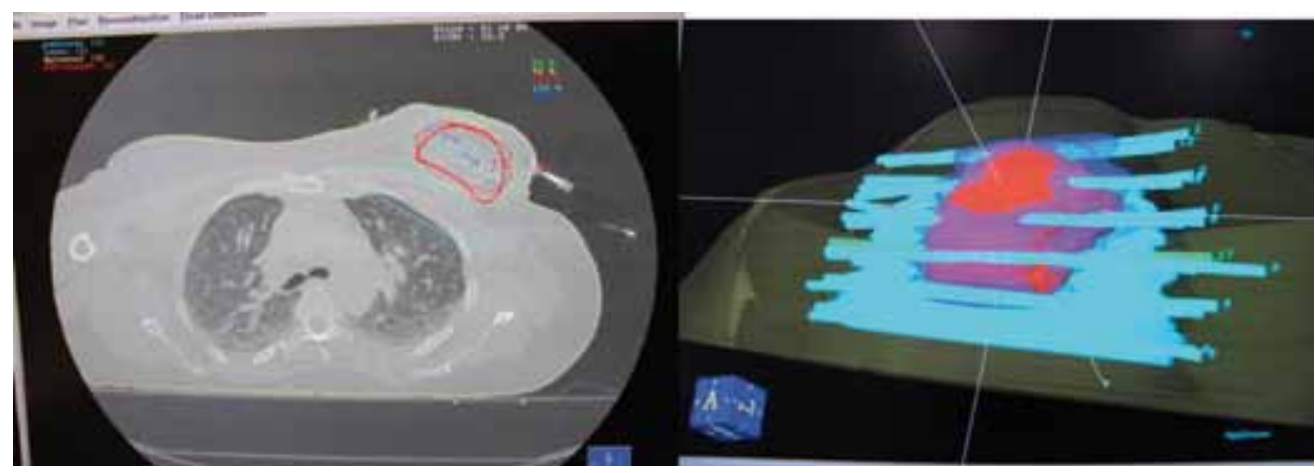

Figura 3. Implante intersticial con tubos plásticos de Sistema Comfort (Nucletron®) en cáncer de mama. Planificación optimizada con tomografía computarizada.

\section{CÁNCER DE CABEZA Y CUELLO}

El tratamiento con braquiterapia exclusiva ha sido de elección en los tumores tempranos de labio, lengua, suelo de boca, mucosa yugal, base de lengua, nasofaringe $^{31}$. Sin embargo, su utilización ha ido progresivamente reduciéndose con la mejora de las técnicas quirúrgicas, que intentan también procedimientos conservadores de órganos y de su función.

En la actualidad, estos procedimientos se realizan sólo en algunos centros con experiencia. Habitualmente forman parte de un tratamiento combinado con RTE y/o cirugía.

Recientemente, el grupo europeo de GEC-ESTRO ha revisado las indicaciones de la braquiterapia en estos tumores ${ }^{32}$. En ellas se recoge la recomendación de incorporar técnicas de imagen como la TC para la delimitación ganglionar y tumoral en relación con el hueso; la RM para detectar la invasión tumoral y de partes blandas y realizar una mejor delineación $3 \mathrm{D}$, y de la PET para conocer el comportamiento biológico tumoral. En ellas se recoge también la técnica de implantación recomendada y las dosis de tratamiento en tumores de labio, mucosa oral, lengua, suelo de boca y orofaringe.

\section{BRAQUITERAPIA PALIATIVA}

La braquiterapia endocavitaria de bronquio y esófago ha mostrado eficacia 
en el control de síntomas con la obstrucción (68\%), el sangrado (71\%) o el dolor $(43 \%)^{33}$. El tratamiento se realiza con la colocación de catéteres plásticos flexibles en estas cavidades a través de endoscopios o incluso de una sonda nasogástrica. En un estudio aleatorizado ${ }^{34}$, la braquiterapia endobronquial fue tan eficaz como la RTE en el control de la obstrucción y el sangrado. En otro ensayo aleatorizado ${ }^{35}$, la braquiterapia endoesofágica fue tan eficaz como las prótesis metálicas en la paliación del dolor y la disfagia.

\section{CONSIDERACIONES FINALES}

La braquiterapia está experimentando un enorme desarrollo en los últimos años. La aparición de nuevos aplicadores de menor tamaño o adaptados a mejores técnicas de imagen han hecho que hoy tenga un gran protagonismo en el tratamiento de pacientes con cáncer de próstata, ginecológico, cáncer de mama, cabeza y cuello... En otros tumores, la combinación de braquiterapia con RTE y/o cirugía muestra resultados interesantes, como en sarcomas especialmente pediátricos, cáncer de recto y ano o tumores torácicos.

La incorporación de técnicas de imagen como la ecografía, la TC, la RM o la PET, permite una mejor definición del tumor y de los órganos sanos a proteger. Pero además, su incorporación a los sistemas de cálculo de dosis durante el mismo implante, con los planificadores en tiempo real, favorece una mejora técnica en el implante y esto empieza a mostrar mejores resultados en la curación de los pacientes con cáncer.

\section{BIBLIOGRAFÍA}

1. Cheron H, Rubens-Duval H. Aperçu sur les resultants de la radiumtherapie des cancers d l'uterus et du vagin. Bull Soc Obst Gynec Par 1913; 2: 419-429.

2. Montegmaggi P, Guerrieri P, Federico M, MorTELLARO G. Clinical Applications of Braquitherapy. En: Halperin EC, Perez CA, Brady L.Principles and prctice of Radiation Oncology Philadelphia: LWW, 2008: 476-535.
3. International commission on Radiation Units. ICRU $\mathrm{n}^{\circ}$ 38: dose an volume specifications for reporting intracavitary therapu in gynecology. Bethesda MD. ICRU, 1985: 1-16.

4. Hoskin PJ, Bownes P. Innovative thecnologies in radiation therapy: brachytherapy. Sem Radiat Oncol 2006; 16: 209-217.

5. Potter R. Image-guided brachytherapy sets benchmarks in advanced radiotherapy. Radiother Oncol 2009; 91: 141-146.

6. MeErtens H, Briot E. Radiophysics. En: Gerbaulet A, Potter R, Mazeron JJ et al. (Edit) GEC ESTRO handbook of brachytherapy. ESTRO Brussels; 2002. p. 23-83.

7. Hilaris Bs, Whitmore WF, Batata Ma, Barzell W. Behavioral patterns of prostate adenocarcinoma following an I125-implant and pelvic node dissection. Int J Radiat Oncol Boil Phys 1977; 2: 631-637.

8. Nag S, Beyer D, Friedland J, Grimm P, Nath R. American Brachytherapy Society (ABS) recommendations for transperineal permanent brachytherapy of prostate cancer. Int $\mathrm{J}$ Radiat Oncol Biol Phys 1999; 44: 789-799.

9. Ash D, Flynn A, Battermann J, de ReiJke T, LAVAGNINI P, BLANK L. ESTRA/EAU Urological Brachytherapy Group; EORTC Radiotherapy Group. ESTRO/EAU/EORTC recommendations on permanent seed implantation for localized prostate cancer. Radiother Oncol 2000; 57: 315-321.

10. Salembier C, Lavagnini P, Nickers P, Mangili P, Rijnders A, Polo A et al. GEC ESTRO PROBATE Group. Tumour and target volumes in permanent prostate brachytherapy: a supplement to the ESTRO/EAU/EORTC recommendations on prostate brachytherapy. Radiother Oncol 2007; 83: 3-10.

11. Stone NN, Potters L, Davis BJ, Ciezki JP, Zelefsky MJ, RoAch M et al. Customized dose prescription for permanent prostate brachytherapy: insights from a multicenter analysis of dosimetry outcomes. Int J Radiat Oncol Biol Phys 2007; 69: 1472-1477.

12. Guedea F, Ellison T, VenselaAr J, Borras JM, Hoskin P, Poetter R et al. Overview of brachytherapy resources in Europe: a survey of patterns of care study for brachytherapy in Europe. Radiother Oncol 2007; 82: 50-54.

13. Stone NN, Potters L, Davis BJ, Ciezki JP, ZeleFSKY MJ, RoACH M et al. Multicenter analysis of effect of high biologic effective dose on biochemical failure and survival outcomes in patients with Gleason score 7-10 prostate cancer treated with permanent prostate 
brachytherapy. Int J Radiat Oncol Biol Phys 2009; 73: 341-346.

14. Ferrer M, Suárez JF, Guedea F, Fernández P, MaCÍAS V, MARIÑo A et al. Multicentric Spanish Group of Clinically Localized Prostate Cancer. Health-related quality of life 2 years after treatment with radical prostatectomy, prostate brachytherapy, or external beam radiotherapy in patients with clinically localized prostate cancer. Int J Radiat Oncol Biol Phys 2008; 72: 421-432.

15. Zelefsky MJ, NedelKa MA, Arican ZL, Yamada Y, CoHEN GN, SHIPPY AM et al. Combined brachytherapy with external beam radiotherapy for localized prostate cancer: reduced morbidity with an intraoperative brachytherapy planning technique and supplemental intensity-modulated radiation therapy. Brachytherapy 2008; 7: 1-6.

16. Galalae RM, Martínez A, Mate T, Mitchell C, Edmundson $\mathrm{G}$, Nuernberg $\mathrm{N}$ et al. Long-term outcome by risk factors using conformal high-dose-rate brachytherapy (HDR-BT) boost with or without neoadjuvant androgen suppression for localized prostate cancer. Int J Radiat Oncol Biol Phys 2004; 58: 10481055.

17. Ghadjar P, Matzinger O, Isaak B, Behrensmeier $F$, Stroux A, Rentsch CA et al. Dose exposure in combined high-dose-rate brachytherapy and intensity modulated radiation therapy in intermediate- and high-risk prostate cancer. Radiother Oncol 2009; 91: 237-242.

18. Gerbaulet A, Potter R, Haie-Meder C. Cervix cancer. En: Gerbaulet A, Potter R, Mazeron JJ et al, editors. GEC ESTRO handbook of brachytherapy. ESTRO Brussels; 2002, pp. 301363.

19. Dimopoulos JC, Schard G, Berger D, Lang S, Goldner G, Helbich T et al. Systematic evaluation of MRI findings in different stages of treatment of cervical cancer: potential of MRI on delineation of target, pathoanatomic structures, and organs at risk. Int J Radiat Oncol Biol Phys 2006; 64: 1380-1388.

20. Haie-Meder C, Pötter R, Van LE, Limbergen E, Briot E, De Brabandere M, Dimopoulus J et al. Recommendations from gynaecological (GYN) GEC-ESTRO working group (I): concepts and terms in 3D image based 3D treatment planning in cervix cancer brachytherapy with emphasis on MRI assessment of GTV and CTV. Radiother Oncol 2005; 74: 235-245.

21. Haie-Meder C, Pötter R, Van Limbergen E, Briot E, De Brabandere M, Dimopoulos J et al. Gynaecological (GYN) GEC-ESTRO Working Group.
Recommendations from gynaecological (GYN) GEC ESTRO working group (II): concepts and terms in 3D image-based treatment planning in cervix cancer brachytherapy-3D dose volume parameters and aspects of 3D image-based anatomy, radiation physics, radiobiology. Radiother Oncol 2006; 78: 67-77.

22. NAG S. Controversies and new developments in gynecologic brachytherapy: image-based intracavitary brachytherapy for cervical carcinoma. Semin Radiat Oncol 2006; 16: 164-167.

23. Pötter R, Dimopoulos J, Georg P, Lang S, WaldHÄUSL C, WAChTER-GeRSTNER N et al. Clinical impact of MRI assisted dose volume adaptation and dose escalation in brachytherapy of locally advanced cervix cancer. Radiother Oncol 2007; 83: 148-155.

24. Poortmans PM, Collette L, Bartelink H, Struikmans H, Van den Bogaert WF, Fourquet A et al. EORTC Radiation Oncology and Breast Cancer Groups. The addition of a boost dose on the primary tumour bed after lumpectomy in breast conserving treatment for breast cancer. A summary of the results of EORTC 22881-10882 "boost versus no boost" trial. Cancer Radiother 2008; 12: 565-570.

25. Polgár C, Major T, Fodor J, Németh G, Orosz $\mathrm{Z}$, SuLYok $\mathrm{Z}$ et al. HDR brachytherapy alone versus whole breast radiotherapy with or without tumor bed boost after breast conserving surgery: seven-year results of a comparative study. Int J Radiat Oncol Biol Phys 2004; 60: 1173-1181.

26. ARTHUR D, VICINI F. Accelerated partial breast irradiation as part of breast conserving therapy. J Clin Oncol 2005; 23: 1726-1735.

27. Polgár C, Fodor J, Major T, Németh G, Lövey K, OROSZ Z et al. Breast-conserving treatment with partial or whole breast irradiation for low-risk invasive breast carcinoma-5-year results of a randomized trial. Int J Radiat Oncol Biol Phys 2007; 69: 694-702.

28. NSABP B-39/RTOG 0413 protocol document. National surgical adjuvant breast and bowel project. Available from: https:// members. nsabp.pitt.edu/B39_Protocol.pdf. Consultado el 10 de julio de 2009 .

29. Groupe Européen de Curietherapie-European Society for Therapeutic Radiology and Oncology Trial Website. Available from: http:// www.apbi.uni-erlangen.de/outline/outline. html. Consultado el 10 de julio de 2009.

30. Jonathan B. Strauss, Adam Dickler. Accelerated partial breast irradiation utilizing balloon brachytherapy techniques. Radiother Oncol 2009; 91: 157-165. 
31. Gerbaulet A, Mazeron JJ. Head and neck cancer (nasopharynx excluded): general aspects. En: Gerbaulet A, Potter R, Mazeron JJ et al, editors. GEC ESTRO handbook of brachytherapy. ESTRO Brussels; 2002. p. 221225 .

32. Mazeron JJ, Ardiet JM, Haie-Méder C, Kovács G, Levendaq P, Peiffert D et al. GEC-ESTRO recommendations for brachytherapy for head and neck squamous cell carcinoma. Radiother Oncol 2009; 91: 150-156.

33. Gaumitz M, Ellerbroek N, Komaki R, Putnam JB JR, Ryan MB, DeCaro L et al. High dose endobronquial irradiation in recurrent broncho- genic carcinoma. Int J Radiat Oncol Biol Phys 1992; 23: 397-400.

34. Stout R, Barber P, Burt P, Hopwood P, Swindell $\mathrm{R}$, HodgetTs $\mathrm{J}$ et al. Clinical and quality of life outcomes in the first United Kingdom randomized trial of endobronchial brachytherapy vs external beam radiotherapy in the palliative treatment of inoperable non small cell lung cancer. Radiother Oncol 56: 323-327, 2000.

35. Shenfine J, McNamee P, Steen N, Bond J, Griffin SM. A pragmatic randomized controlled trial of the cost-effectiveness of palliative therapies for patients with inoperable oesophageal cancer. Health Technol Assess 2005; 9: 1-100. 
